\title{
Leptomeningeal metastasis from early glottic laryngeal cancer: A case report
}

\author{
ZHENYU PAN $^{1 *}$, GUOZI YANG ${ }^{1 *}$, LIMEI QU $^{2}$, TINGTING YUAN $^{3}$, XIAOCHUAN PANG ${ }^{4}$, \\ YONGXIANG WANG ${ }^{4}$, WEIYAN SHI ${ }^{1}$ and LIHUA DONG ${ }^{1}$ \\ Departments of ${ }^{1}$ Radiotherapy, ${ }^{2}$ Pathology, ${ }^{3}$ Radiology and ${ }^{4}$ Clinical Laboratory, \\ Norman Bethune First Hospital of Jilin University, Changchun, Jilin 130021, P.R. China
}

Received October 21, 2014; Accepted July 28, 2015

DOI: $10.3892 / 01.2015 .3643$

\begin{abstract}
The present study reports the case of a 53-year-old man with leptomeningeal metastasis from early glottic laryngeal cancer. The patient had been diagnosed with squamous cell carcinoma of the glottic larynx 9 years previously. The current symptoms included a recurring headache that had persisted for 1 month and vomiting for 1 week. A magnetic resonance imaging scan of the head revealed multiple enhancing lesions in the brain and multiple line-like enhancements in the brain fold. Computed tomography scans of the head, neck, chest and abdomen showed no nodular lesions. Cytological examination of the cerebral spinal fluid (CSF) revealed malignant cells with a scattered distribution pattern. The patient received intra-CSF methotrexate chemotherapy concurrent with whole-brain radiotherapy, which relieved the neurological symptoms. To the best of our knowledge, this is the first case of cytologically-confirmed LM from early glottic laryngeal cancer.
\end{abstract}

\section{Introduction}

Laryngeal cancer accounts for the majority of head and neck cancers diagnosed worldwide (1). The spread of laryngeal cancer follows the anatomical structure of the larynx, which is divided into the glottis (true vocal cords, and anterior and posterior commissures), the supraglottis (epiglottis, arytenoid folds and false cords), and the subglottis (1). The majority of laryngeal cancers originate in the glottis and are classified as squamous cell carcinomas (SCCs). Laryngeal cancer commonly spreads via direct extension to adjacent structures

Correspondence to: Dr Lihua Dong, Department of Radiotherapy, Norman Bethune First Hospital of Jilin University, 71 Xinmin Street, Changchun, Jilin 130021, P.R. China

E-mail:dlh@jlu.edu.cn

${ }^{*}$ Contributed equally

Key words: leptomeningeal metastasis, glottic larynx cancer, magnetic resonance imaging, cytological examination through metastasis to regional cervical lymph nodes. Distant metastases, however, are not commonly observed in laryngeal malignancies $(2,3)$.

Leptomeningeal metastasis (LM), a lethal complication of certain cancers, is the metastatic tumor cell invasion of the leptomeninges (arachnoid and pia mater) of the meninx and arachnoid spaces (4). To date, only a few cases of LM due to head and neck cancer have been reported (5-8). Moreover, a literature search revealed no reports of confirmed LM from glottic laryngeal cancer. The present study reports a case of LM from glottic laryngeal cancer and reviews the corresponding literature to gain insight into this rare event.

\section{Case report}

A 53-year-old male was admitted to Norman Bethune First Hospital on February $13^{\text {th }}, 2014$ with an ongoing headache that had persisted for 1 month and the occurrence of regular vomiting for 1 week. The patient had no history of chronic disease or excessive alcohol use, but reported a 20-year smoking history. The patient had experienced hoarseness 9 years previously and had sought medical treatment. Laryngoscopy detected SCC of the glottic larynx. A laryngeal cleft and right vocal cord resection was performed. Tumor cells were polygonal, and exhibited scattered distribution; additionally, keratinization was observed within the cells. The post-operative pathological examination showed SCC (moderately-differentiated) of tumor stage T1N0M0 (9). The patient was administered prophylactic radiotherapy due to tumor invasion into the anterior commissure of the larynx. Radiotherapy to the tumor bed and preventive radiotherapy to the cervical lymph node (50 Gy, 25 times/5 weeks) was administered. The patient experienced no evident side-effects from this treatment, but exhibited hoarseness post-surgery.

During the present admission period, the physical examination indicated symptoms of drowsiness, hypologia and simple communication abilities. In addition, no pyramid sign and no evident cranial nerve sign were observed. Routine hepatic and renal function tests, and blood and coagulation tests were normal. The patient was negative for hepatitis B, hepatitis $\mathrm{C}$, syphilis and human immunodeficiency virus. Tumor marker levels for cancer antigen (CA)125, CA15, CA72-4, neuron-specific enolase, cytokeratin 19 fragment 21-1, CA19-9, 

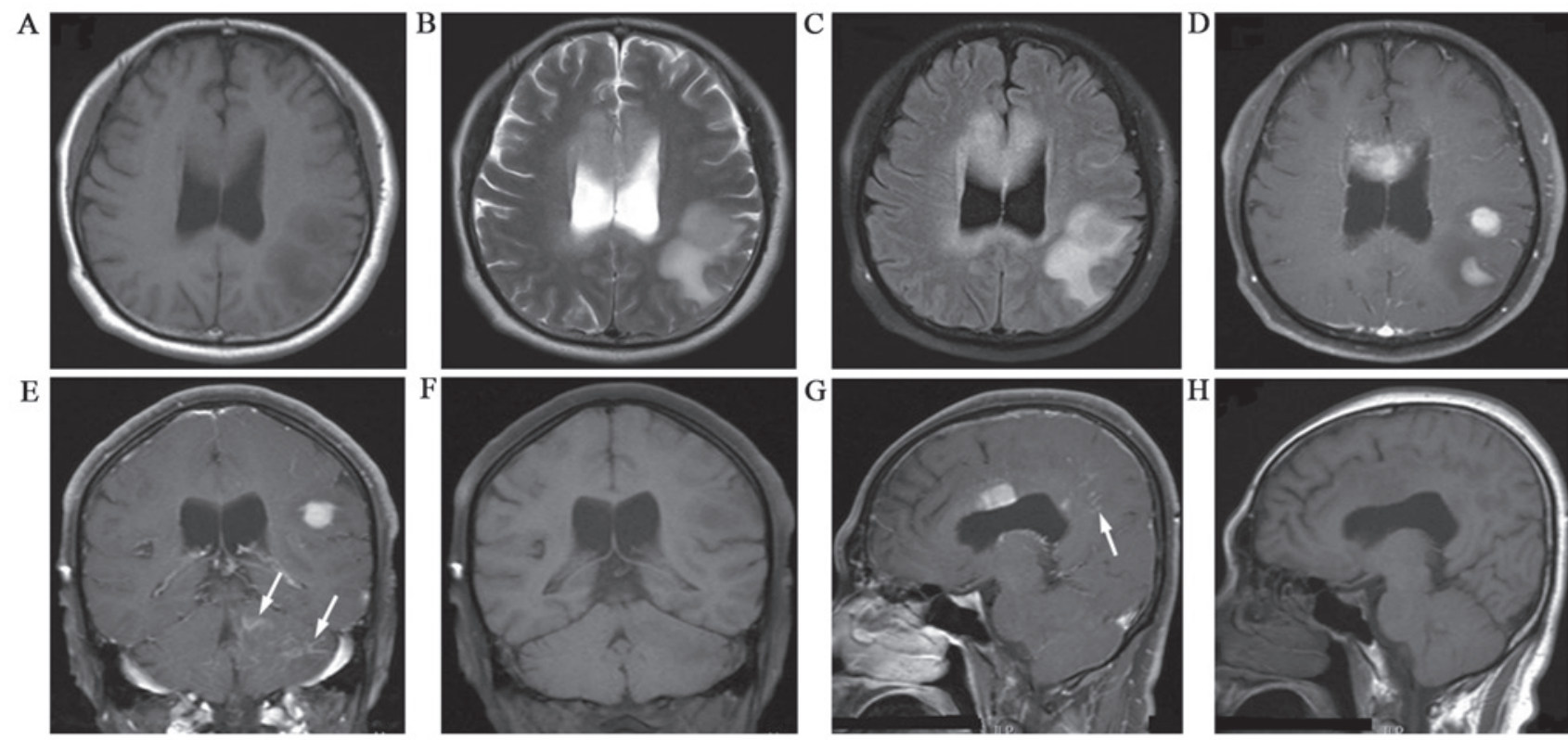

Figure 1. Initial 3.0 magnetic resonance imaging scan of the head. (A) Transverse, (F) coronal and (H) sagittal T1-weighted images; (B) T2-weighted image; (C) fluid-attenuated inversion recovery; and (D, E and G) contrast enhancement images used for diagnosis and to establish a clinical therapy. The imaging examination showed multiple lesions in the occipital lobe, parietal lobe, lateral ventricles and corpus callosum of the brain. Line-like enhancement in the brain fold of the cerebrum and cerebellum was also visualized (arrows).

A

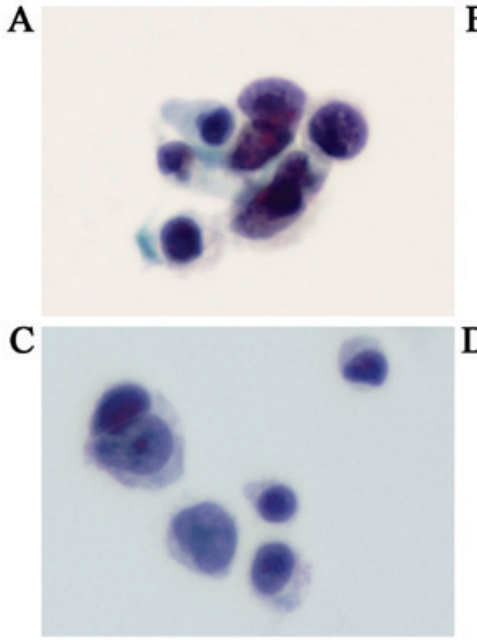

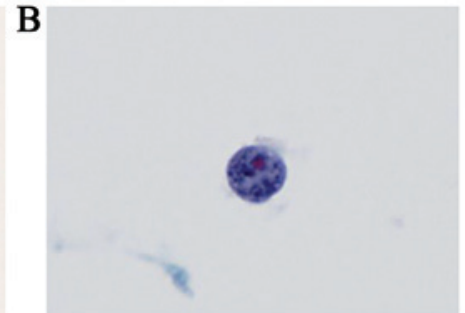

D

Figure 2. Cytological examination of the cerebral spinal fluid (liquid-based technology, Papanicolaou staining; magnification, x400). The examination revealed (A and B) a scattered distribution of malignant cells with irregular cell sizes, pleomorphism, greatly increased nuclear-to-cytoplasmic ratios, intensely-stained cytoplasm and round, red-stained nucleoli in certain nuclei. (C and D) Certain malignant cells were large in size, with multiple nuclei and nucleoli.

$\alpha$-fetoprotein and carcinoembryonic antigen were all normal. Computed tomography (CT) scans of the head and neck, and chest and abdomen indicated that no nodular lesions were present. A magnetic resonance imaging (MRI) scan of the head revealed multiple enhancing lesions in the brain and multiple line-like enhancements in the brain fold (Fig. 1). The patient was therefore diagnosed with LM from glottic laryngeal cancer.

At 3 days post-admission, the patient exhibited aconuresis, lethargy and unconsciousness, indicating rapid disease progression. The Glasgow coma score (GCS) was 11-12 points (normal score, 15) and the Karnofsky performance status (KPS) score was 20 points (normal score, 100 points) $(10,11)$. A lumbar puncture was performed, which resulted in the following (normal ranges presented in brackets following measured value): Intracranial pressure, $310 \mathrm{~mm} \mathrm{H}_{2} \mathrm{O}\left(80-180 \mathrm{~mm} \mathrm{H}_{2} \mathrm{O}\right)$; protein level, $1.57 \mathrm{~g} / \mathrm{l}$ (0.15-0.45 g/l); glucose level, $3.91 \mathrm{mmol} / 1$ (2.3-4.1 mmol/l); chlorine level, $111.7 \mathrm{mmol} / \mathrm{l}(119-129 \mathrm{mmol} / \mathrm{l})$; white blood cell count, 149 $\times 10^{6} / 1\left(0-8 \times 10^{6} / 1\right)$; and red blood cell count, $0 \times 10^{6} / 1\left(0 \times 10^{6} / 1\right)$. Antibodies against viral infection of the cerebrospinal fluid (CSF), Microspironema pallidum or Mycobacterium tuberculosis were not present, and bacterial smears, Mycobacterium tuberculosis smears, and Ink staining were all negative. A cytological examination of the CSF was performed via liquid-based technology (ThinPrep TCT2000; Hologic, Bedford, MA, USA), and the malignant cells were observed via Papanicolaou staining (Fig. 2). The 

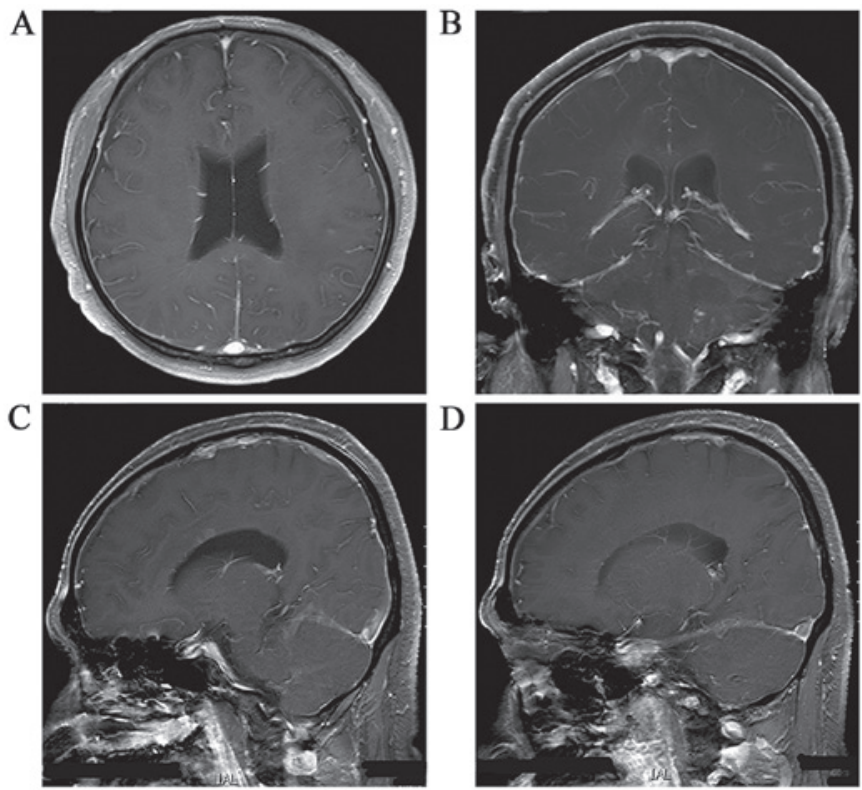

Figure 3. Post-therapy 3.0 magnetic resonance imaging scan of the head (contrast enhancement scanning). The imaging examination showed that the original lesions in the brain and the line-like enhancement in the brain fold were nearly gone. (A) Transverse, (B) coronal and (C and D) sagittal view.

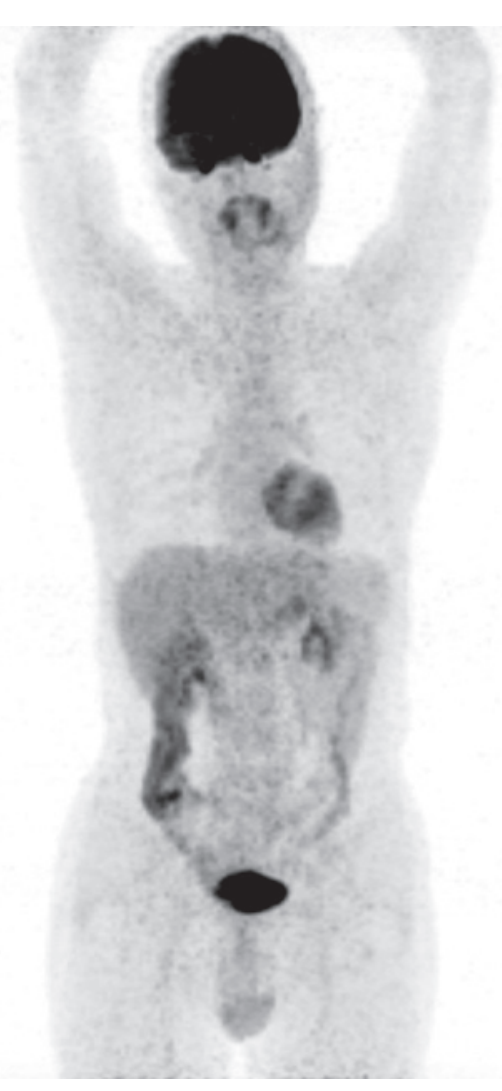

Figure 4. Positron emission tomography/computed tomography scan at 3 months post-treatment demonstrating no abnormal fluorodeoxyglucose uptake.

presence of metastatic tumor cells in the CSF confirmed the diagnosis of LM from glottic laryngeal cancer.

The patient was immediately administered nutritional support (fat emulsion, $250 \mathrm{ml}$; amino acids, $250 \mathrm{ml}$; glucose,
$1,000 \mathrm{ml} ; 10 \%$ kalium chloratum, $20 \mathrm{ml}$; multivitamins; pantoprazole, $40 \mathrm{mg}$; once per day for 3 weeks), intracranial pressure reduction therapy $(20 \%$ mannitol, $250 \mathrm{ml}$; glycerol fructose, $250 \mathrm{ml}$; twice per day for 3 weeks), glucocorticoid therapy (dexamethasone, $10 \mathrm{mg}$ per day, then $5 \mathrm{mg}$ after 5 days, continued until radiation therapy was accomplished) and intrathecal chemotherapy (15 mg methotrexate and $5 \mathrm{mg}$ dexamethasone). The patient regained consciousness 5 days later, with relief from the vomiting and the ability to perform simple verbal communication. The GCS was $12-14$ points. The patient then underwent radiotherapy of the whole brain and skull base (linear accelerator $6 \mathrm{MV}$ X-ray, 2 Gy/day) concurrent with intrathecal chemotherapy once a week (same regimen as aforementioned). The patient's headache was resolved 2 weeks later and the vomiting had completely stopped. However, routine blood tests detected a reduction in the white blood cell count $\left(2.89 \times 10^{9} / 1\right)$, and lower extremity numbness accompanied the intrathecal chemotherapy. The myelosuppression and lower extremity numbness were alleviated once the patient received recombinant human granulocyte colony-stimulating factor (150 $\mu \mathrm{g}$ per day, days 1-5) and calcium folinate (100 mg twice per day via intravenous injection for 1 week). Following 40 Gy (2 Gy/day) of whole-brain radiotherapy for 4 weeks and 6 intrathecal chemotherapy treatments $(15 \mathrm{mg}$ methotrexate; $5 \mathrm{mg}$ dexamethasone; once a week), the headache was alleviated and the patient was able to remain conscious. The GCS was 15 points and the KPS score was 60 points. An MRI scan of the head showed that the original lesions in the brain were almost completely gone (Fig. 3). CT scans of the neck, chest and abdomen exhibited no nodular lesions. Further treatment was suspended. At 3 months post-treatment, positron emission tomography/CT was performed and no abnormalities were found (Fig. 4). It has currently been 9 months since the diagnosis of LM and the patient is disease-free with ongoing follow-up examinations.

\section{Discussion}

Laryngeal cancer is the most common cancer of the head and neck (12). The incidence of laryngeal tumors closely correlates with smoking and drinking (13). A retrospective study (1) reported that the most frequent sites of distant metastases in patients with SCC of the head and neck (HNSCC) were the lungs, bones and liver, while metastasis to the brain was less common. In addition, $21.8 \%$ of patients were diagnosed with HNSCC at an early stage (T1-2). Relative to other malignancies, the distant metastases of HNSCC often develop slowly, with only $16 \%$ of patients presenting with distant metastases at the time of diagnosis. In the remaining $84 \%$ of patients, distant metastases were observed after a median time of 381 days (1). Thus, the time from the diagnosis until the occurrence of distant metastases of HNSCC is longer than for other malignancies, and local early lesions also have a high risk of distant metastasis.

LM is an extremely rare but devastating form of metastatic spread. Only 6 HNSCC cases (1) with LM have been reported (carcinoma of paranasal sinuses, carcinoma of ethmoid sinus, carcinoma of tongue, carcinoma of lip, carcinoma of the palatine tonsil and carcinoma of the supraglottic larynx), and only 1 case of SCC of the supraglottic larynx with LM has been 
described (5-8). However, the primary tumor site of the previously reported HNSCC cases differed from that observed in the present study. To the best of our knowledge, there have been no previous studies in the literature describing LM secondary to early glottic larynx cancer. LM can be a fatal complication of malignancies, and results from metastatic infiltration of the leptomeninges (arachnoid and pia mater) of the meninx and arachnoid spaces by malignant cells originating from an extrameningeal primary tumor site (4). The most common methods for diagnosing LM are neuroimaging and the cytological demonstration of malignant cells in the CSF, with the latter being the gold standard for the diagnosis of this condition (4). For the present case, the MRI scan of the head showed line-like enhancement in the brain fold of the cerebrum and cerebellum. Cytological examination of the CSF was also performed and showed a scattered or clustered distribution of malignant cells. The cells were irregular in size and pleomorphic, with greatly increased nuclear-to-cytoplasmic ratios, intensely-stained cytoplasm and round, red-stained nucleoli in certain nuclei. All of these features are classic malignant characteristics of metastatic cells in the CSF. Due to the patient's critical condition, the CSF examination was limited and other immunocytochemistry examinations were not performed. However, according to the varied malignant characteristics of the malignant cells that were detected in the CSF, the patient could be definitively diagnosed with LM. The patient had developed glottic laryngeal cancer 9 years previously, however, CT scans of the neck, chest and abdomen showed no nodular lesions except those of the nervous system. Furthermore, no lesions were found on CT scans after 7 months of treatment.

Accordingly, the patient was diagnosed with LM from glottic laryngeal cancer. LM-directed treatment is currently palliative and its goal is to improve or stabilize the patient's neurological status, improve their quality of life and prolonging their survival time (4). Systemic and intra-CSF chemotherapy and involved-field radiotherapy are common and effective treatments for this disease, and methotrexate is the most commonly utilized intra-CSF chemotherapy drug in the treatment of LM from solid tumors $(4,14)$. In a previous report of a case in which the LM arose from a laryngeal SCC, the patient was treated with intra-CSF methotrexate chemotherapy and palliative radiotherapy. Although the patient's pain improved, the lower limb weakness worsened and the patient succumbed 3 weeks after completing the radiotherapy (6). In the present case, the patient was administered intra-CSF chemotherapy first. When the patient's central nervous system symptoms, such as the unconsciousness state and vomiting, were relieved, the patient immediately received concurrent whole-brain radiotherapy and intra-CSF chemotherapy. The patient's symptoms were rapidly alleviated, and the GCS and KPS scores increased. An MRI scan of the head demonstrated that the original lesions in the brain were almost completely gone, while CT scans of the chest and abdomen showed no nodular lesions. Furthermore, no severe adverse reactions of the central nervous system occurred during or after treatment, indicating the effectiveness of the therapy.
In conclusion, this is the first report of a rare case of LM from early glottic laryngeal cancer. Clinicians should be aware of the possibility of LM in patients presenting with an appropriate constellation of signs and symptoms. Moreover, it is important to report that, in the present study, the treatment of LM from early glottic laryngeal cancer with intra-CSF methotrexate chemotherapy concurrent with whole-brain radiotherapy was effective.

\section{Acknowledgements}

The authors would like to thank Medjaden Bioscience Ltd., (Hong Kong, China) for assisting in the preparation of this manuscript.

\section{References}

1. Spector ME, Chinn SB, Rosko AJ, Worden FP, Ward PD, Divi V, McLean SA, Moyer JS, Prince ME, Wolf GT, et al: Diagnostic modalities for distant metastasis in head and neck squamous cell carcinoma: Are we changing life expectancy? Laryngoscope 122: 1507-1511, 2012

2. Furtado de Araújo Neto VJ, Cernea CR, Aparecido Dedivitis R, Furtado de Araújo Filho VJ, Fabiano Palazzo J and Garcia Brandão L: Cervical metastasis on level IV in laryngeal cancer. Acta Otorhinolaryngol Ital 34: 15-18, 2014.

3. Gallegos JF, Fuentes A, Arroyo C, Minauro G, Hernández M and Arias $\mathrm{H}$ : Laryngeal function as node metastasis predictor in patients with cancer of the larynx. Gac Med Mex 146: 175-178, 2010.

4. Le Rhun E, Taillibert S and Chamberlain MC: Carcinomatous meningitis: Leptomeningeal metastases in solid tumors. Surg Neurol Int 4 (Suppl 4): S265-S288, 2013.

5. Redman BG, Tapazoglou E and Al-Sarraf M: Meningeal carcinomatosis in head and neck cancer. Report of six cases and review of the literature. Cancer 58: 2656-2661, 1986.

6. Thompson SR, Veness MJ, Morgan GJ, Shannon J and Kench JG: Leptomeningeal carcinomatosis from squamous cell carcinoma of the supraglottic larynx. Australas Radiol 47: 325-330, 2003.

7. Banerjee TK and Gottschalk PG: Unusual manifestations of multiple cranial nerve palsies and mandibular metastasis in a patient with squamous cell carcinoma of the lip. Cancer 53: 346-348, 1984.

8. Biswal BM, Goyal M, Prasad RR, Lal P, Sharma R, Mohanti BK and Khader J: Leptomeningeal carcinomatosis from carcinoma of the palatine tonsil. Australas Radiol 42: 66-68, 1998.

9. Pfister DG, Spencer S, Brizel DM, Burtness B, Busse PM, Caudell JJ, Cmelak AJ, Colevas AD, Dunphy F, Eisele DW, et al: Head and neck cancers, version 2.2014. Clinical practice guidelines in oncology. J Natl Compr Canc Netw 12: 1454-1487, 2014.

10. Teasdale G and Jennett B: Assessment of coma and impaired consciousness. A practical scale. Lancet 2: 81-84, 1974.

11. Karnofsky DA and Burchenal JH: The clinical evaluation of chemotherapeutic agents in cancer. In: Evaluation of Chemotherapeutic Agents. MacLeod CM (ed). Columbia University Press, Columbia, p196, 1949.

12. Wenig BM: Squamous cell carcinoma of the upper aerodigestive tract: Precursors and problematic variants. Mod Pathol 15: 229-254, 2002.

13. Igissinov N, Zatoskikh V, Moore MA, Igissinov S, Toulebaeyev R, Mustafina M, Valieva S, Aldiyarova G, Bukeyeva $Z$ and Venglovskiy A: Epidemiological evaluation of laryngeal cancer incidence in Kazakhstan for the years 1999-2009. Asian Pac J Cancer Prev 14: 3969-3974, 2013.

14. Pan Z, Yang G, Yuan T, Pang X, Wang Y, Qu L and Dong L: Leptomeningeal metastasis from hepatocellular carcinoma with other unusual metastases: A case report. BMC Cancer 14: 399, 2014. 